\title{
STUDIES ON CHLOROPHYCEAN DIVERSITY OF MALHARA POND OF BHADRAWATI, DIST- CHANDRAPUR (M.S.), INDIA.
}

\section{Harney, N.V.1 , A.A. Dhamani ${ }^{2}$ and R.J. Andrew ${ }^{3}$}

${ }_{1}^{1}$ Deptt. of Zoology, Nilkanthrao Shinde Science and Arts College, Bhadrawati Distt. Chandrapur-442902

2 P.G. Deptt. of Zoology, N.H. College, Bramhapuri, Distt. Chandrapur-441206 3 P.G. Deptt. of Zoology, Hislop College, Nagpur-440001

Email of corresponding author: narendra_harney2008@rediffmail.com

\section{ABSTRACT:}

The phytoplankton in aquatic ecosysytems is an important biological indicator of the water quality. While phytoplankton are major primary producers and the basis of the food chain in open water, some species on the other hand can be harmful to human and other organisms by releasing toxic subtances into the water. Phytoplankton plays an important role a primary producers in fresh water ecosystem. Phytoplankton are the primary producers, which form the base of an autotrophic food chain. They are of great importance as source of live food for zooplankton and fishes. The present paper describes the diversity of Chlorophyceae of Malhara pond, located near the Bhadrawati town of Chandrapur district. Qualitative and quantitative analysis of Chlorophyceae community was undertaken on monthly basis from October 2005 to September 2007. A total of 24 chlorophycean species were identified during the period of Oct. 2005 to Sep. 2006, while a total of 19 chlorophyceaen species were identified from Oct. 2006 to Sep 2007. In Malhara pond Vorticella sp. was dominant followed by Volvox sp., Closterium chodati, Netrium digitus, Euastrophis richteri and Pendiatrum tetras and the present study demonstrated that the Chlorophyceae was maximum during the summer season and minimum during the monsoon season. The seasonal fluctuation of this group is discussed in the light of recent literature.

Key words- Malhara pond, Chlorophycean diversity, Seasonal variation.

\section{INTRODUCTION:}

Phytoplanktons are the autotrophic component of the plankton community. Since they obtain energy through the process of photosynthesis and must therefore live in the well-lit surface layer (termed the euphotic zone) of a water body. Phytoplanktons are the base of most of the lakes food web and fish 
productions is linked to phytoplanktons production (Ryder et al., 1974). The present investigation has been undertaken to study the qualitative and quantitative analysis of chlorophyceaen community at the Malhara pond located near Bhadrawati town of Chandrapur district.

\section{MATERIAL AND METHODS:}

The Malhara pond is situated on the west side, four miles away from the town and it is located about $206 \mathrm{~m}$. above the mean sea level and is at $79^{\circ} 06^{\prime}$ $48^{\prime \prime} \mathrm{E}$ longitude and $20^{\circ} 06^{\prime} 48^{\prime \prime} \mathrm{N}$ latitude. The samples were collected in the morning hours between 8.30 to 10.30 a.m. 50 Lt. of water sample was filtrated through the plankton net made of bolting silk number 25 with mesh size 64 limes. The collected samples were allowed to settle down by adding Lugol's iodine. Normally, sedimentation requires $24 \mathrm{hrs}$. After which supernatant was removed and concentrate was made up to $50 \mathrm{ml}$ depending the number of plankton and preserved in $5 \%$ formalin for further studies.

The quantitative study of chlorophyceae was done by Sedgwick - Rafter cell method, the concentrated sample was shaken and immediately one drop of sample was taken on a clear micro side with the help of a standard dropper, the whole drop was then carefully covered with the cover glass and observed. Identification up to genera and whenever possible up to species level was classified according to keys given by Prescott (1954), Edmondson (1959), Sehgal (1983), Adoni ( 1985) and APHA (1985).

\section{RESULT AND DISCUSSION:}

Phytoplanktons are ecologically significant as they trap radiant energy of sunlight and convert it to chemical energy i.e. organic material. The role of phytoplanktons in managing bioenergetics, as bioindicators and as purifiers of polluted aquatic habitat is well known. 
During the year 2005-06, 24 species were recorded among which Vorticella sp. (244 no./lit.) is dominent followed by Volvox sp. (193 no./lit.), Coelastrum chodati (32 no./lit.), Netrium digitus (26 no./lit.), Euastropsis richteri (24 no./lit.), Pediastrum tetras (21 no./lit.), Micrasterias pinnatifida (19 no./lit.), Closerium leibleimi (17 no./lit.), Chlamydomonas sp. (16 no./lit.), Goniochloris sp. (15 no./lit.), Ankistrodesmus sp. (15 no./lit.), Staurastrum sp. (11 no./lit.) and Pleurodiscus sp. (11 no./lit.), Chara sp. (9 no./lit.), Chlorococcum humicola (8 no./lit.) and Hydrodictyon (8 no./lit.), Cylindrospermum sp. (7 no./lit.) and Oedogonium sp. (7 no./lit.) and Chlorella sp. (6 no./lit.) and Nitella sp. (6 no./lit.).

During the year 2006-07, 19 species were recorded among which Volvox sp. (159 no./lit.) is dominant followed by Ankistrodesmus sp. (163 no./lit.), Vorticella sp. (30 no./lit.), Cosmarium granatum (23 no./lit.), Gloeocystis gigas (20 no./lit.), Microsterias pinnatifida (14 no./lit.), Staurastrum sp. (14 no./lit.), Pleurodiscus sp. (14 no./lit.), Netrium digitus (13 no./lit.), Spirogyra sp. (12 no./lit.), Oedogonium sp. (11 no./lit.) and Chara sp. (11 no./lit) Chlorococcum humicola (10 no./lit.), Pediastrum tetras (10 no./lit.), Chlorella sp. (10 no./lit.), Troschiscia pachyderma (8 no./lit.), Hydrodictyon sp. (8 no./lit.), Chlamydomonas sp. (7 no./lit.) and Cylindrospermum sp. (6 no./lit.).

Chloarophyceae was the most dominant group among all the planktons. The dominance of Chlorophyceae was also recorded by Sakhare and Joshi (2002) in Yeldari reservoir, Nanded district, Maharashtra and Jayabhaye et al., (2007) in Parola dam, Hingoli district, Maharashtra. Somani and Pejawar (2003) reported 14 genera of Chlorophyceae in Masunda, Thane, Maharashtra. Kumawat and Jawale (2003) recorded 14 genera belonging to Chlorophyceae from a freshwater pond at Dharmapuri in Beed district, Maharashtra and 59 genera of phytoplankton from a fishpond at Anjale. Pawar et al., (2006) reported 26 species of Chlorophyceae and observed Ankistrodesmus falcatus as 
a dominant species in Chloropyceae in Petwadas dam of Kandhar of Nanded district, Maharashtra. Tiwari and Chouhan (2006) reported 34 species of Chloropyceae in Kitham lake, Agra, Uttar Pradesh. Waghmare and Mali (2007) reported 10 species of Chlorophyceae in a minor irrigation dam of Kalamnuri, District Hingoli, Maharashtra. Mukherjee et al., (2010) reported 8 species of chlorophyceae in a polluted eutrophic lake, Ranchi. Anjayan, K.V. and Parameshwara Naik, T. (2011) reported 9 genera in lentic water bodies of Bhadrawati taluk, Shimoga District, Karnataka. Shinde et al., (2012) recorded the 15 genera in Harsool-Savangi dam, Aurangabad, India.

Among the different species in Malhara pond Vorticella sp. was dominant followed by Volvox sp., Closterium chodati, Netrium digitus, Euastrophis richteri and Pendiatrum tetras.

In the present investigation, Chlorophyceae was maximum during the summer season and minimum during the monsoon season. Reddy (1984) found a positive corelationship between temperature and green algae in Tungabhadra river, Karnataka. Kumawat and Jawale (2003) observed dominance of Chlorophyceae in the month of April. Khapekar and Deshpande (2007) reported similar observation during the assesement of Naik lake, Nagpur, Maharashtra. Factors such as high temperature, low nitrate and bright sunlight are favorable for the population of green algae (Rao, 1955).

Jayabhaye et al., (2007) reported maximum Chloropyceae population during the summer and minimum during the rainy season in Parola dam, Hingoli, Maharashtra. Factors such as high temperature, low nitrate and a bright sunlight are favorable conditions for high population of green algae (Rodhe, 1948). In the present investigation, maximum Chlorophyceae during the summer is due to high temperature. 
Table No. 1 : Monthly variation of Chlorophyceaen diversity in Malhara Pond During 2005-06

\begin{tabular}{|c|c|c|c|c|c|c|c|c|c|c|c|c|c|c|}
\hline \multirow{2}{*}{$\begin{array}{l}\text { Sr. } \\
\text { No. }\end{array}$} & \multirow{2}{*}{ Components } & \multicolumn{4}{|c|}{ Winter } & \multicolumn{4}{|c|}{ Summer } & \multicolumn{4}{|c|}{ Monsoon } & \multirow{2}{*}{ Total } \\
\hline & & Oct & Nov & Dec & Jan & Feb & Mar & Apr & May & Jun & Jul & Aug & Sep & \\
\hline 1 & Ankistrodesmus $s p$ & 0 & 0 & 0 & 0 & 4 & 11 & 0 & 0 & 0 & 0 & 0 & 0 & 15 \\
\hline 2 & Chara sp. & 1 & 1 & 1 & 6 & 0 & 0 & 0 & 0 & 0 & 0 & 0 & 0 & 9 \\
\hline 3 & Chlamydomonas sp. & 6 & 4 & 1 & 5 & 0 & 0 & 0 & 0 & 0 & 0 & 0 & 0 & 16 \\
\hline 4 & Chlorella $s p$. & 1 & 1 & 1 & 1 & 1 & 1 & 0 & 0 & 0 & 0 & 0 & 0 & 6 \\
\hline 5 & $\begin{array}{l}\text { Chlorococcum humicola } \\
\text { sp. }\end{array}$ & 0 & 0 & 0 & 1 & 3 & 4 & 0 & 0 & 0 & 0 & 0 & 0 & 8 \\
\hline 6 & Closterium leibleimi sp. & 0 & 0 & 2 & 4 & 6 & 5 & 0 & 0 & 0 & 0 & 0 & 0 & 17 \\
\hline 7 & Coelastrum chodati & 6 & 6 & 2 & 1 & 1 & 5 & 0 & 0 & 0 & 0 & 0 & 11 & 32 \\
\hline 8 & Cosmarium granatum & 1 & 3 & 2 & 5 & 5 & 5 & 0 & 0 & 0 & 0 & 0 & 0 & 21 \\
\hline 9 & Cylindrospermum sp. & 1 & 1 & 1 & 4 & 0 & 0 & 0 & 0 & 0 & 0 & 0 & 0 & 7 \\
\hline 10 & Euastropsis richteri & 6 & 4 & 6 & 1 & 2 & 5 & 0 & 0 & 0 & 0 & 0 & 0 & 24 \\
\hline 11 & Glaeocystis gigas & 0 & 0 & 6 & 3 & 5 & 1 & 4 & 0 & 0 & 0 & 0 & 0 & 19 \\
\hline 12 & Goniochloris sp. & 5 & 5 & 1 & 0 & 0 & 0 & 0 & 0 & 0 & 0 & 0 & 4 & 15 \\
\hline 13 & Hydrodictyon $s p$. & 1 & 1 & 2 & 4 & 0 & 0 & 0 & 0 & 0 & 0 & 0 & 0 & 8 \\
\hline 14 & Micrasterias pinnatifida & 1 & 1 & 2 & 4 & 5 & 6 & 0 & 0 & 0 & 0 & 0 & 0 & 19 \\
\hline 15 & Netrium digitus & 6 & 7 & 2 & 4 & 4 & 3 & 0 & 0 & 0 & 0 & 0 & 0 & 26 \\
\hline 16 & Nitella $s p$. & 1 & 4 & 1 & 0 & 0 & 0 & 0 & 0 & 0 & 0 & 0 & 0 & 6 \\
\hline 17 & Oedogonium sp. & 1 & 1 & 2 & 3 & 0 & 0 & 0 & 0 & 0 & 0 & 0 & 0 & 7 \\
\hline 18 & Pediastrum tetras & 1 & 1 & 17 & 1 & 1 & 0 & 0 & 0 & 0 & 0 & 0 & 0 & 21 \\
\hline 19 & Pleurodiscus sp. & 1 & 3 & 0 & 0 & 0 & 0 & 0 & 0 & 0 & 0 & 0 & 7 & ממ \\
\hline 20 & Spirogyra sp. & 0 & 0 & 0 & 3 & 6 & 5 & 0 & 0 & 0 & 0 & 0 & 0 & 14 \\
\hline
\end{tabular}


International Journal of Researches in Biosciences, Agriculture \& Technology

September 2013

Issue-1, Volume-1
ISSN No. (Online):

2347-517X

\begin{tabular}{|l|l|c|c|c|c|c|c|c|c|c|c|c|c|c|}
21 & Staurastrum sp. & 0 & 0 & 2 & 4 & 3 & 2 & 0 & 0 & 0 & 0 & 0 & 0 & 11 \\
\hline 22 & Trochiscia pachyderma & 0 & 0 & 6 & 2 & 1 & 5 & 0 & 0 & 0 & 0 & 0 & 0 & 14 \\
\hline 23 & Volvox $s p$. & 6 & 21 & 18 & 7 & 21 & 6 & 21 & 0 & 0 & 0 & 67 & 26 & 193 \\
\hline 24 & Vorticella $s p$. & 6 & 1 & 3 & 5 & 6 & 0 & 51 & 72 & 52 & 48 & 0 & 0 & 244 \\
\hline & Total & 51 & 65 & 78 & 68 & 74 & 64 & 76 & 72 & 52 & 48 & 67 & 48 & 763 \\
\hline
\end{tabular}

Table No. 2 : Monthly variation of Chlorophyceaen diversity in Malhara Pond During 2006-07

\begin{tabular}{|c|c|c|c|c|c|c|c|c|c|c|c|c|c|c|}
\hline \multirow{2}{*}{$\begin{array}{l}\text { Sr. } \\
\text { No. }\end{array}$} & \multirow{2}{*}{ Components } & \multicolumn{4}{|c|}{ Winter } & \multicolumn{4}{|c|}{ Summer } & \multicolumn{4}{|c|}{ Monsoon } & \multirow{2}{*}{ Total } \\
\hline & & Oct & Nov & Dec & Jan & Feb & Mar & Apr & May & Jun & Jul & Aug & Sep & \\
\hline 1 & $\begin{array}{l}\text { Ankistrodesmus } \\
s p \text {. }\end{array}$ & 0 & 0 & 0 & 0 & 12 & 11 & 3 & 68 & 47 & 22 & 0 & 0 & 163 \\
\hline 2 & Chara sp. & 0 & 0 & 0 & 0 & 0 & 0 & 0 & 0 & 0 & 0 & 0 & 11 & 11 \\
\hline 3 & $\begin{array}{l}\text { Chlamydomonas } \\
\text { sp. }\end{array}$ & 2 & 1 & 3 & 1 & 0 & 0 & 0 & 0 & 0 & 0 & 0 & 0 & 7 \\
\hline 4 & Chlorella sp. & 1 & 1 & 1 & 1 & 1 & 1 & 1 & 1 & 1 & 1 & 0 & 0 & 10 \\
\hline 5 & $\begin{array}{l}\text { Chlorococcum } \\
\text { humicola }\end{array}$ & 0 & 0 & 0 & 2 & 1 & 4 & 3 & 0 & 0 & 0 & 0 & 0 & 10 \\
\hline 6 & $\begin{array}{l}\text { Cosmarium } \\
\text { granatum }\end{array}$ & 2 & 1 & 1 & 6 & 2 & 1 & 10 & 0 & 0 & 0 & 0 & 0 & 23 \\
\hline 7 & $\begin{array}{l}\text { Cylindrospermum } \\
\text { sp. }\end{array}$ & 2 & 1 & 2 & 1 & 0 & 0 & 0 & 0 & 0 & 0 & 0 & 0 & 6 \\
\hline 8 & Gloeocystis gigas & 0 & 0 & 6 & 3 & 1 & 4 & 6 & 0 & 0 & 0 & 0 & 0 & 20 \\
\hline 9 & Hydrodictyon $s p$ & 3 & 1 & 3 & 1 & 0 & 0 & 0 & 0 & 0 & 0 & 0 & 0 & 8 \\
\hline 10 & $\begin{array}{l}\text { Micrasterias } \\
\text { pinnatifida }\end{array}$ & 1 & 1 & 1 & 2 & 4 & 3 & 2 & 0 & 0 & 0 & 0 & 0 & 14 \\
\hline 11 & Netrium digitus & 1 & 1 & 1 & 1 & 4 & 3 & 2 & 0 & 0 & 0 & 0 & 0 & 13 \\
\hline 12 & Oedogonium sp. & 2 & 1 & 3 & 5 & 0 & 0 & 0 & 0 & 0 & 0 & 0 & 0 & 11 \\
\hline 13 & Pediastrum tetras & 1 & 6 & 1 & 1 & 1 & 0 & 0 & 0 & 0 & 0 & 0 & 0 & 10 \\
\hline 14 & $\begin{array}{l}\text { Pleurodiscus } \\
\text { species }\end{array}$ & 2 & 1 & 0 & 0 & 0 & 0 & 0 & 0 & 0 & 0 & 0 & 11 & 14 \\
\hline 15 & Spirogyra sp. & 0 & 0 & 0 & 1 & 2 & 1 & 8 & 0 & 0 & 0 & 0 & 0 & 12 \\
\hline 16 & Staurastrum $s p$ & 0 & 0 & 1 & 2 & 3 & 6 & 2 & 0 & 0 & 0 & 0 & 0 & 14 \\
\hline
\end{tabular}


International Journal of Researches in

September 2013

ISSN No. (Online):

Biosciences, Agriculture \& Technology

Issue-1, Volume-1

2347-517X

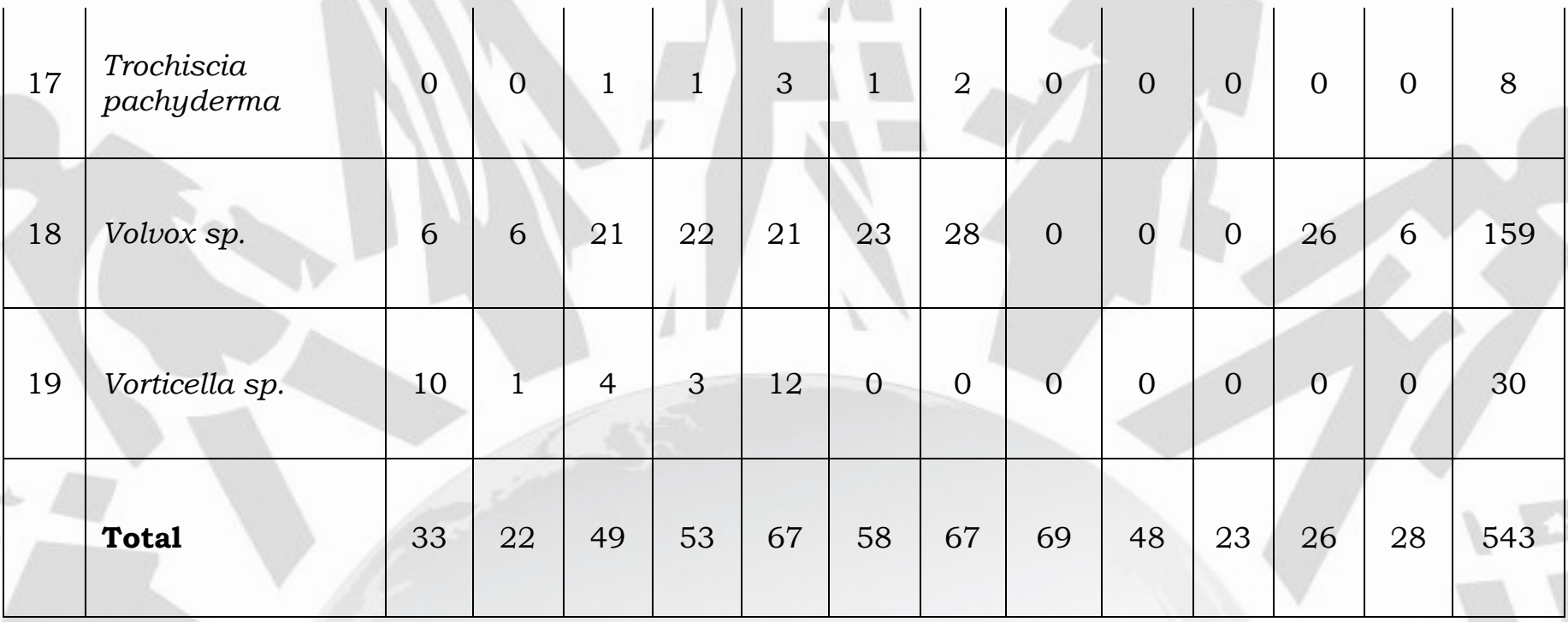

Table No. 3 : Seasonal variation of Chlorophycea in Malhara Pond During year 2005-06

\begin{tabular}{|c|c|c|c|c|c|c|c|}
\hline \multirow{2}{*}{$\begin{array}{l}\text { Sr. } \\
\text { No. }\end{array}$} & \multirow{2}{*}{ Components } & \multicolumn{2}{|c|}{ Winter } & \multicolumn{3}{|c|}{ Summer } & Monsoon \\
\hline & & Mean & S.D. & Mean & & S.D. & Mean \\
\hline 1 & Chlorophyceae & 65.500 & \pm 9.657 & 71.500 & \pm & 4.555 & $53.750 \pm 7.822$ \\
\hline & Total & 65.500 & \pm 9.657 & 71.500 & \pm & 4.555 & $53.750 \pm 7.822$ \\
\hline
\end{tabular}

Table No. 4. :Seasonal variation of Chlorophyceae in Malhara Pond During year 2006-07

\begin{tabular}{|c|c|c|c|c|c|c|}
\hline \multirow{2}{*}{\begin{tabular}{|l|} 
Sr. \\
No
\end{tabular}} & \multirow{2}{*}{ Components } & \multicolumn{3}{|c|}{ Winter } & Summer & Monsoon \\
\hline & & Mean & & S.D. & Mean & Mean \\
\hline 1 & Chlorophyceae & 39.25 & \pm & 12.457 & $65.250 \pm 4.265$ & $31.250 \pm 9.833$ \\
\hline & Total & 39.25 & \pm & 12.457 & $65.250 \pm 4.265$ & $31.250 \pm 9.833$ \\
\hline
\end{tabular}




\section{REFERENCES:}

Adoni, A.D. (1985) Studies on microbiology of Sagar lake, Ph.D. Thesis, Sagar University Sagar, Madhya Pradesh. pp. 243.

Ajayan, K.V. \& Parameshwara Naik, T. (2011) Seasonal temperature and pollution indicator of phytoplankton in lentic water bodies of Bhadrawati taluk, Shimoga District, Karnataka. J. Eco. \& Fisheries. 4(1): 71-76.

APHA (1985) Standard methods of examination of water \& waste water. American Public Health Association, 19th Ed. Inc. New York, pp.1170.

Edmondson, W.T. (Ed.) (1959) Freshwater Biology. Edward and Whipple, 2 $2^{\text {nd }}$ Ed., John Wiley Sons Ins. New York, pp 1248.

Jayabhaye, U.M., V.R. Madlapure \& B.S. Salve (2007) Phytoplankton diversity of Parola Dam, Hingoli, Maharashtra. J. Aqua. Biol. 22(2): 27-32.

Khapekar, R.R. \& Deshpande, S.V. (2007) Phytoplankton composition and assessment of pollution starts of Naik lake, Nagpur, Maharashtra. J. Aqua. Biol. 22(2): 21-26.

Kumawat, D.A. \& Jawale, A.K. (2003) Phytoplonkton of a fish pond at Anjale, Maharashtra. Eco. Env. \& Cons. 9(3): 411-415.

Kumawat, D.A. \& Jawale, A.K. (2003) Phytoplonkton ecology of a fish pond at Anjale reservoir, Jalgaon, Maharashtra. J. Aqua. Biol. 18(1): 9-13.

Mukharjee, B., M. Nivedita \& D. Mukharjee (2010) Plankton diversity and dynamics in a polluted eutrophic lake, Ranchi. J. Env. Biol. 31(5): 827839. 
Pawar, S.K., J.S.Pulle \& K.M. Shendage (2006) The study on phytoplankton of Pethwadaj Dam, Taluka Kandhar, District Nanded, Maharashtra, . J. Aqua. Biol. 21(1): 1-6.

Prescott, G.W. (1954) The fresh- water algae. W M C Brown company. Dubuque, USA.

Rao, C.B. (1955) On the distribution of algae in the group of six small ponds II. Algal productivity. J. Ecol. 43: 291-308.

Rodhe, W. (1948) Environmental requirement of a fresh water plankton algae. VII Iron as a limiting factor for growth, Symp. Bio. Ops. 10: 104-117.

Ryder, R.A., Kerr, S.R., Loftus, K.H. \& Register, H.A. (1974) The morphoedaphic index, a fish yield estimator review and evaluation. $J$. fisheries Res. Canada. 31: 663-668.

Sakhre, V.B. \& JoshI, P.K. (2002) Ecology of Palas Nilegaon Reservoir in Osmanabad, Maharashtra. J. Aqua. Biol. 17(2): 17-22.

Sehgal, K. L. (1983) Planktonic Copepod of Fresh Water System. ITCR print, New Delhi.

Shinde, S.E., T.S. Pathan \& Sonawane, D.L. (2012) Seasonal variation and biodiversity of phytoplankton in Harsool-Savangi dam, Aurangabad, India. J. Env. Biol. 33: 643-647.

Somani, V.U. \& Pejawar, M.K. (2003) Rotifer diversity in lake Masunda, Thane, Maharashtra. J. Aqua. Biol. 18(1): 23-27.

Tiwari, A. \& Chouhan, S.V.S. (2006) Seasonal phytoplanktonic diversity of Kitham lake, Agra. J. Aqua. Biol. 27 (1): 35-38.

Waghmare, V.N. \& Mali, R. P. (2007) The study of phytoplankton of Kalammuri minor irrigation dam, Kalamnuri district, Hingoli, Maharashtra. J. Aqua. Biol. 22 (2): 59-62. 